\title{
Homeopatia: Desinformação e Preconceito no Ensino Médico
}

\author{
Homeopathy: Lack of Information and \\ Prejudice in Medical Teaching
}

Marcus Zulian Teixeira ${ }^{1}$

PALAVRAS-CHAVE:

- Educação Médica;

- Homeopatia;

- Medicina Complementar;

- Atitude;

- Currículo.

KEY-WORDS:

- Education, Medical;

- Homeopathy;

- Complementary Medicine;

- Attitude;

- Curriculum.

Recebido em: 23/02/2006

Reencaminhado em: 18/10/2006

Aprovado em: 11/01/2007

\section{RESUMO}

Embora a homeopatia seja uma especialidade médica reconhecida pelo Conselho Federal de Medicina desde 1980, com pressupostos científicos estabelecidos, aplicação clínica diversa, projetos nas áreas de pesquisa básica e clínica, oferecida nos serviços públicos de saúde e com iniciativas de ensino na graduação médica, a desinformação sobre esta peculiar racionalidade se encontra arraigada na cultura médica. Este estudo objetivou mensurar a desinformação quanto aos pressupostos homeopáticos existente entre estudantes de Medicina participantes do $33^{\circ}$ Encontro Científico de Estudantes de Medicina (São Paulo, 2003). Foi aplicado um questionário auto-responsivo no início de uma atividade didática, no qual o conhecimento foi mensurado. Os respondentes consideravam como "prerrogativas da homeopatia": o tratamento natural (18\%), o efeito placebo (14\%) e o aspecto místico-religioso (4,5\%); "indicações do tratamento homeopático" se restringiram às doenças crônicas (52\%) ou psicossomáticas (18\%); "inexistência de fundamentação científica" pela pesquisa básica (21\%) ou clínica (29\%); "morosidade na resposta terapêutica" (57\%); e "isenção de efeitos colaterais" no uso inadequado do medicamento homeopático (71\%). Perante outros aspectos, $43 \%$ dos estudantes não reconheciam a homeopatia como uma "especialidade médica"; a totalidade ignorava que ela estivesse "disponível em serviços públicos de saúde"; $64 \%$ desconheciam sua "inclusão no currículo de algumas faculdades de Medicina"; e todos os alunos se mostraram "bastante interessados em aprendê-la", na forma de disciplina obrigatória (64\%) ou optativa (36\%). Sugere-se que a informação acerca dos aspectos fundamentais da homeopatia seja transmitida nos primeiros anos da graduação médica.

\begin{abstract}
Although Homeopathy is a medical specialty recognized by the Federal Counsel of Medicine since 1980, with established scientific purposes, diversified clinical applications, projects in basic and clinical research, available at the public health services and with initiatives towards inclusion in the curriculum of higher medical education, the lack of information about this peculiar practice is still deeply rooted in the medical culture. The aim of this study was to measure the lack of information about the premises of homoeopathy existing among medicine students participating in the 33rd Scientific Meeting of Medicine Students (33- Encontro Científico de Estudantes de Medicina) - São Paulo, 2003. The knowledge was measured by means of a self-applicable questionnaire distributed in the beginning of the didactic activity. The students answering the questionnaire considered "prerogatives of homeopathy" natural treatment (18\%), placebo effect (14\%), and the mystic-religious aspect (4.5\%); "indications for homeopathic treatment" were restricted to chronic diseases (52\%) or psychosomatic diseases (18\%); "inexistence of a scientific basis" was affirmed by basic research (21\%) and clinical research (29\%), "slow therapeutic response" by 57\%, and "absence of adverse effects" in case of inadequate use of homeopathic
\end{abstract}


drugs by 71\%. Above all, 43\% of the students did not recognize homeopathy as a "medical specialty", all of them ignored that it was "available at the public health services" and $64 \%$ did not know about its "inclusion in the curriculum of some Medical Schools". Considering that all students demonstrated "great interest in studying it", be it as compulsory (64\%) or as optional discipline (36\%), it is suggested that information about the fundamental aspects of homeopathy should be transmitted in the first years of medical graduation.

\section{INTRODUÇÃO}

Tanto no Brasil quanto em outros países, aumentam a cada ano o interesse e a procura da população por "Práticas Não-Convencionais em Saúde" (PNCS), destacando-se a fitoterapia, a homeopatia e a acupuntura ${ }^{1-5}$.

Para suprir esta demanda de mercado, ocupada em diversos países por terapeutas não-médicos, a classe médica vêm demonstrando interesse crescente no aprendizado de $\mathrm{PNCS}^{6-9}$, predispondo as escolas de Medicina a incorporarem, na última década, o ensino destas modalidades terapêuticas ao currículo fundamental da graduação.

Levantamento realizado em 1996 no Reino Unido ${ }^{10}$ mostrou que $23 \%$ das faculdades de Medicina haviam incorporado ao currículo disciplinas que ministravam conceitos básicos sobre as diversas formas de PNCS. Em 1999, 40\% das escolas médicas da União Européia ${ }^{11}$ ofereciam cursos em PNCS. No período de 1997-1998, uma pesquisa em 117 escolas médicas americanas ${ }^{12}$ mostrou que $64 \%$ ensinavam PNCS, evidenciando-se aumento desta estimativa em levantamentos recentes ${ }^{13}$. Em 1998, pesquisa realizada junto às escolas médicas do Canadá ${ }^{14}$ evidenciou que $81 \%$ delas apresentavam tópicos de PNCS em seu currículo. Um levantamento em 80 escolas médicas japonesas ${ }^{15}$, no período de 1998-1999, mostrou que 20\% ensinavam PNCS, num total de 25 cursos, prevalecendo o ensino da acupuntura. Em geral, estas disciplinas são ministradas de forma superficial, agrupando-se uma grande variedade de práticas distintas num mesmo módulo, restando uma carga horária insuficiente para o entendimento destas peculiares e complexas abordagens terapêuticas (1-2 horas para cada modalidade).

Apesar deste movimento globalizante, ainda é insuficiente o número de escolas de Medicina que incorporaram ao currículo fundamental o ensino sistemático de PNCS, privando a maioria dos médicos do efetivo esclarecimento acerca dos preceitos fundamentais e das evidências científicas que respaldam tais terapêuticas, ferramentas indispensáveis à orientação adequada das indicações e dos riscos destas práticas médicas a seus pacientes ${ }^{16-18}$.
Além da desinformação médica, o afastamento destas racionalidades do meio acadêmico dificulta o desenvolvimento de pesquisas voltadas à fundamentação científica dos pressupostos teóricos e da prática clínica, retardando as perspectivas de maior entendimento dos princípios ortodoxos destas práticas, distintos daqueles empregados pela medicina convencional.

Segundo levantamento realizado pela Fundação Fiocruz e pelo CFM (1996) junto aos médicos brasileiros, a homeopatia, como especialidade principal de atuação, ocupa o $17^{\circ}$ maior contingente de profissionais nas 61 especialidades analisadas 19 .

Apesar de a homeopatia ser reconhecida como uma especialidade médica pelo Conselho Federal de Medicina desde 1980, com aplicação clínica bicentenária, pressupostos científicos estabelecidos, projetos de pesquisa nas áreas básica e clínica, disponibilidade em serviços públicos de saúde e iniciativas de ensino na graduação médica, a desinformação sobre estes aspectos fundamentais gera conceitos distorcidos, que são incorporados à cultura médica brasileira.

Buscando mensurar a informação dos estudantes de $\mathrm{Me}-$ dicina perante estes fundamentos do modelo homeopático, realizamos uma oficina com estudantes de Medicina participantes do $33^{\circ}$ Encontro Científico de Estudantes de Medicina (ECEM, São Paulo, 2003), propondo uma "sistemática educacional breve" que poderá preencher, num primeiro momento, esta lacuna do ensino médico brasileiro.

\section{MÉTODOS}

Um questionário auto-aplicável foi respondido por 18 estudantes de Medicina no início de uma oficina realizada por ocasião do $33^{\circ}$ Encontro Científico de Estudantes de Medicina (2003). Nele, o conhecimento homeopático foi mensurado conforme as seguintes variáveis: tipo de conhecimento e forma de aquisição; pressupostos do modelo homeopático; eficácia terapêutica e principais indicações; pesquisas básica e clínica; morosidade da resposta terapêutica e efeitos adversos; especialidade médica, disponibilidade em serviços públicos e ensino nas escolas médicas; importância e interesse no aprendizado.

Fizeram parte da oficina 18 alunos dos primeiros quatro anos da graduação, de nove faculdades de Medicina de diversos estados brasileiros (PR, SP, MG, DF, GO, AL, PE, RN).

\section{RESULTADOS}

Os respondentes consideravam como "pressupostos da homeopatia" o tratamento natural (18\%), o efeito placebo $(14 \%)$ e o aspecto místico-religioso $(4,5 \%)$. Os pressupostos 
homeopáticos foram relatados por uma parcela dos estudantes (princípio da similitude: $23 \%$; experimentação no homem são: 4,5\%; emprego de ultradiluições: $36 \%$ ).

Em relação aos "tipos de doenças" passíveis de tratamento, os respondentes apontaram que a homeopatia é "eficaz" apenas nas doenças crônicas (52\%) e nas doenças psicossomáticas $(18 \%)$.

A maioria dos estudantes se colocou positivamente quanto à "existência de fundamentação científica" para os pressupostos homeopáticos $(78 \%)$ e para o tratamento das doenças (71\%).

Cinqüenta e sete por cento dos estudantes consideraram o tratamento homeopático "demorado" ("em doses homeopáticas"), assim como $71 \%$ isentaram os medicamentos de "efeitos adversos" ("se não fizer bem, mal não faz").

A totalidade dos alunos desconhecia a disponibilidade da homeopatia nos "serviços públicos de saúde", sendo que 43\% não sabiam que era uma "especialidade médica" e 64\% não tinham conhecimento da sua "inclusão no currículo da graduação" de algumas faculdades de Medicina brasileiras.

Todos os alunos referiram "bastante interesse" em conhecer melhor a homeopatia, com $64 \%$ julgando importante sua incorporação no currículo das faculdades de Medicina como "disciplina obrigatória" e 36\% como "disciplina optativa".

\section{DISCUSSÃO}

Observamos um nível insuficiente de informação dos estudantes de Medicina sobre os "pressupostos homeopáticos", com resultados semelhantes aos de pesquisas realizadas em outros países com estudantes de Medicina ${ }^{20}$, médicos-residentes $^{21}$ e médicos ${ }^{9}$, as quais buscaram avaliar o nível de conhecimento sobre diversas PNCS, fator que contribui para o desconhecimento destas práticas terapêuticas no meio médico.

Como os estudantes referiram no questionário, as principais indicações de encaminhamento de pacientes para tratamento homeopático por colegas de outras especialidades se restringem a "doenças crônicas" que não responderam aos tratamentos convencionais. Mesmo assim, estes casos de extrema cronicidade, desvitalizados por inúmeras agressões medicamentosas sofridas ao longo de anos, encontram melhora efetiva com o tratamento homeopático prolongado ${ }^{22-24}$, aspecto qualitativo pouco valorizado quando se questiona a eficácia do modelo homeopático.

Respaldando a resposta da maioria dos estudantes entrevistados, que atribuíram fundamentação científica ao tratamento das doenças ("eficácia clínica da homeopatia"), apesar do baixo grau de conhecimento prévio, uma metanálise de 12 levantamentos sobre as atitudes de médicos convencionais perante as PNCS mostrou que eles geralmente creditam moderada eficácia às mesmas ${ }^{6}$.

Demonstrando esta mesma dinâmica, pesquisa realizada recentemente com médicos de diversas especialidades residentes do município de São Paulo evidenciou que as PNCS são prevalentes no cotidiano do médico paulistano, sendo que $87,6 \%$ referiram perceber demanda por parte dos pacientes. Metade dos médicos mostrou atitude positiva em relação às PNCS; 52\% endossam ou prescrevem algum tipo; 20\% referem treinamento; e 13\% informam ser provedores de pelo menos uma modalidade de PNCS. Ao serem indagados sobre a influência das PNCS, 61,5\% opinaram que há influência positiva no resultado terapêutico do paciente; $42,8 \%$ acham que essas práticas alteram positivamente o trabalho do médico; $61,9 \%$ entendem que causam alguma ação sobre o resultado terapêutico. As modalidades que os entrevistados mais referiram conhecer foram: acupuntura, homeopatia, terapias em grupo, dietas alternativas e massagem. Noventa e um por cento concordaram em que é importante o médico ter algum conhecimento em PNCS; 69,5\% discordaram de que devam ser combatidas pela classe médica; $85,4 \%$ disseram que devem ser utilizadas somente se forem científicas. Quanto ao treinamento, mais de $60 \%$ acharam importante recebê-lo, inclusive na formação médica ${ }^{25}$.

A afirmação de que "o tratamento homeopático é demorado" pode ser entendida e justificada em vista do tempo gasto no processo de individualização do medicamento perante cada paciente (segundo a totalidade sintomática característica), que demanda, por meio de consultas periódicas, um processo de ajuste nas hipóteses diagnósticas medicamentosas levantadas e/ou nas doses e nas potências do medicamento corretamente indicado ${ }^{26,27}$.

A comparação com "tratamentos naturais", erroneamente isentados de efeitos adversos ou colaterais pela crença popular, induz à falsa conclusão de que a homeopatia "se não fizer bem, mal não faz". Acrescente-se a isto a prática empírica de muitos colegas que misturam medicamentos, doses e potências homeopáticas diversas, sem qualquer critério metodológico ou científico, muitas vezes associando-os indiscriminadamente a outras práticas não-convencionais, como se a terapêutica homeopática fosse uma panacéia. Descrita em todas as épocas pela maioria dos clássicos homeopatas, a aplicação insensata de medicamentos, doses e potências homeopáticas pode causar agravações, patogenesias, supressões e outras manifestações indesejáveis, contrapondo a inverossimilhança da inocuidade do tratamento homeopático ${ }^{28-30}$.

O posicionamento favorável dos estudantes à inclusão da disciplina de homeopatia no currículo médico fundamental 
encontra correspondência em levantamentos realizados com estudantes de Medicina de diversos países, os quais pesquisaram o interesse no aprendizado de $\mathrm{PNCS}^{31-36}$.

As opiniões dos estudantes de Medicina citadas neste trabalho reiteram as atitudes dos acadêmicos da Faculdade de Medicina da Universidade de São Paulo (FMUSP) perante a homeopatia e a acupuntura em pesquisa que utilizou um questionário auto-aplicável com perguntas sobre interesse no aprendizado e na forma de ensino; nível de conhecimento e forma de aquisição; experiência e eficácia da terapêutica em si próprios ou em pessoas próximas; principais indicações e eficácia geral; oferecimento e integração nos serviços públicos de saúde.

Na média das duas disciplinas, mais de $85 \%$ dos estudantes consideraram que elas deveriam estar inseridas no currículo da graduação das escolas médicas de forma opcional (72\%) ou obrigatória (19\%), com 56\% dos entrevistados mostrandose bastante interessados no aprendizado. Apesar de nenhum ou pouco conhecimento no assunto (76\%), 67\% creditavam algum grau de eficácia às mesmas, tendo como principais indicações as doenças crônicas, isoladamente (37\%) ou englobando também as doenças agudas (29\%). Ao redor de 35\% dos acadêmicos foram favoráveis ao oferecimento ambulatorial de ambas as especialidades nos serviços públicos de saúde, enquanto a média de $34 \%$ defendia a oferta destes tratamentos também em hospitais, com $60 \%$ acreditando na possibilidade de integração com a medicina convencional ${ }^{37}$.

\section{CONCLUSÕES}

Apesar de se tratar de amostragem intencional, não representativa do coletivo de estudantes de Medicina, os resultados encontrados na pesquisa com um grupo de estudantes de Medicina presentes no $33^{\circ}$ Encontro Científico de Estudantes de Medicina foram semelhantes aos de outros levantamentos realizados com estudantes de Medicina em diversos países ${ }^{38-46}$.

Os acadêmicos se mostraram interessados em aprender os fundamentos da homeopatia, posicionando-se favoravelmente à inclusão da disciplina no currículo da graduação. Apresentando conhecimento prévio insuficiente, observaram e relataram a eficácia do tratamento, valorizando o emprego destas terapêuticas em doenças crônicas.

Como pudemos observar, a falta de informação dos estudantes sobre os preceitos homeopáticos básicos é clara, ainda mais se considerarmos que o interesse em participar da oficina demonstra um viés de favorecimento da homeopatia.

A mudança de atitude dos alunos ficou evidente após a aquisição de um conhecimento cognitivo mínimo (quatro horas de aula), demonstrando a importância da informação dos preceitos homeopáticos básicos na educação dos futuros médicos.

Para dirimir as falsas idéias que pesam sobre a homeopatia, sugerimos aos responsáveis pelo currículo da graduação médica que o ensinamento dos aspectos fundamentais desta especialidade médica seja transmitido, de forma breve e obrigatória, nos primeiros anos das faculdades de Medicina, independentemente do aprofundamento posterior em disciplinas mais abrangentes.

Exemplificando esta integração de atividades relacionadas à homeopatia na Faculdade de Medicina da Universidade de São Paulo (FMUSP), a adoção desta "modalidade didática breve" (2-4 horas-aula) poderia ser implementada na disciplina "Introdução à Medicina e Suas Especialidades - MCM1674", oferecida pelo Departamento de Clínica Médica aos alunos do primeiro ano da graduação como disciplina obrigatória.

Após esta breve introdução, os alunos interessados em aprofundar o estudo dos conceitos homeopáticos poderiam se inscrever na disciplina optativa "Fundamentos da Homeopatia - MCM0773", oferecida aos estudantes dos $3^{\circ}$ e $4^{\circ}$ anos da graduação desde 2002, com carga horária suficiente (75 horas-aula) para prepará-los para a participação na "Liga Acadêmica de Homeopatia". Nesta, estudarão, mais especificamente, os aspectos relacionados à clínica e à terapêutica homeopáticas, acompanhando pacientes e desenvolvendo atividades de ensino e pesquisa em homeopatia.

\section{REFERÊNCIAS}

1. Eisenberg DM, et al. Unconventional medicine in the United States: prevalence, costs and patterns use. N Eng J Med. 1993; 328: 246-252.

2. Fisher P, Ward A. Complementary medicine in Europe. Br Med J. 1994; 309: 107-111.

3. Eisenberg DM, et al. Trends in alternative medicine use in the United States, 1990-1997: Results of a follow-up national survey. JAMA. 1998; 280: 1569-1575.

4. Burg MA, Hatch RL, Neims AH. Lifetime use of alternative therapy: a study of Florida residents. South Med J. 1998; 91: 1126-1131.

5. Teixeira MZ, Lin CA, Martins MA. O ensino de práticas não-convencionais em saúde nas faculdades de medicina: panorama mundial e perspectivas brasileiras. Rev Bras Educ Med. 2004; 28: 51-60.

6. Ernst E, Resch KL, Whie A. Complementary medicine: what physicians think of it. Arch Intern Med. 1995; 155: 24052408. 
7. Sikand A, Laken M. Pediatricians' experience with and attitudes toward complementary/alternative medicine. Arch Pediatr Adolesc Med. 1998; 152: 1059-1064.

8. Pirotta MV, Cohen MM, Kotsirilos V, Farish SJ. Complementary therapies: have they become accepted in general practice? Med J Aust. 2000; 172: 105-109.

9. Corbin Winslow L, Shapiro H. Physicians want education about complementary and alternative medicine to enhance communication with their patients. Arch Intern Med. 2002; 162: 1176-1181.

10. Morgan D, Glanville H, Mars S, Nathanson V. Education and training in complementary and alternative medicine: a postal survey of UK universites, medical schools and faculties of nurse education. Comp Ther Med. 1998; 6: 6470 .

11. Barberis L, de Toni E, Schiavone M, Zicca A, Ghio R. Unconventional medicine teaching at the Universities of the European Union. J Altern Complement Med. 2001; 7: 337-343.

12. Wetzel MS, Eisenberg DM, Kaptchuck TJ. Courses involving complementary and alternative medicine at U.S. medical schools. JAMA. 1998; 280: 784-787.

13. Brokaw JJ, Tunnicliff G, Raess BU, Saxon DW. The teaching of complementary and alternative medicine in U.S. medical schools: a survey of course directors. Acad Med. 2002; 77: 876-881.

14. Ruedy J, Kaufman DM, MacLeod H. Alternative and complementary medicine in Canadian medical schools: a survey. Can Med Assoc J. 1999; 160: 816-817.

15. Tsuruoka K, Tsuruoka Y, Kajii E. Complementary medicine education in Japanese medical schools: a survey. Complement Ther Med. 2001; 9: 28-33.

16. White AR, Mitchell A, Ernst E. Familiarization with complementary medicine: report of a new course for primary care physicians. J Altern Complement Med. 1996; 2: 307-314.

17. Straus SE. Complementary and alternative medicine: challenges and opportunities for American medicine. Acad Med. 2000; 75: 572-573.

18. Konefal J. The challenge of educating physicians about complementary and alternative medicine. Acad Med 2002;77:847-850.

19. Machado MH, Avila C, Oliveira ES, Sertã F, Lozana JS, Pinto LF. Perfil dos médicos no Brasil. Rio de Janeiro: FIOCRUZ/ CFM-MS/ PNUD, 1996. Disponível em: <http:/ /www.ensp.fiocruz.br/rorehs/producao/perfil_medicos/>.
20. Rampes H, Sharples F, Maragh S, Fisher P. Introducing complementary medicine into the medical curriculum. J R Soc Med. 1997; 90: 19-22.

21. Kemper KJ, Vincent EC, Scardapane JN. Teaching an integrated approach to complementary, alternative, and mainstream therapies for children: a curriculum evaluation. J Altern Complement Med. 1999; 5: 261-268.

22. Linde K, Clausius N, Ramirez G, et al. Are the clinical effects of homeopathy placebo effects? A meta-analysis of placebocontrolled trials. Lancet. 1997; 350: 834-843.

23. Linde K, Melchart D. Randomized controlled trials of individualized homeopathy: a state-of-the-art review. J Altern Complement Med. 1998; 4: 371-388.

24. Jonas WB, Kaptchuk TJ, Linde K. A critical overview of homeopathy. Ann Intern Med. 2003; 138: 393-399.

25. Akiyama K. Práticas não-convencionais em medicina no Município de São Paulo [Dissertação]. São Paulo: Faculdade de Medicina da Universidade de São Paulo; 2004.

26. Teixeira MZ. Protocolo para pesquisa clínica em homeopatia: aspectos fundamentais. [Protocol for clinical research in homeopathy: basic aspects]. Diagn Tratamento. $2001 ; 6$ : 11-18.

27. Teixeira MZ. Será mesmo o fim da homeopatia? Diagn Tratamento. 2006; 11(1): 61-63.

28. Teixeira MZ. Estudo sobre doses e potências homeopáticas [Study about homeopathic doses and potencies]. Rev Homeopatia (SP) 1995; 60: 3-23.

29. Teixeira MZ. Agravação e prognóstico em homeopatia [Aggravation and prognosis in homeopathy]. Rev Homeopatia (SP) 1997; 62: 27-68.

30. Teixeira MZ. Agravação homeopática: uma síntese para a prática. Rev Homeopatia AMHB 1998; 2: 87-95.

31. Halliday J, Taylor M, Jenkins A, Reilly D. Medical students and complementary medicine. Compl Ther Med. 1993; 1 supl: 32-33.

32. Andritzky W. Medical students and alternative medicine a survey. Gesundheitswesen. 1995; 57: 345-348.

33. Haltenhof $H$, Schumm A, Bühler KE. Komplementärmedizin im Urteil von Studierenden der Medizin: Eine Befragung in Vorklinik und Klinik. Forschende Komplementärmedizin. 1997; 5: 284-291.

34. Duggan K, Verhoef MJ, Hilsden RJ. First-year medical students and complementary and alternative medicines: attitudes, knowledge and experiences. Ann R Coll Physicians Surg Can. 1999; 32: 157-160. 
35. Derr S, Shaikh U, Rosen A, Guadagnino P. Medical students' attitudes toward, knowledge of, and experience with complementary nedicine therapies. Acad Med. 1998; 73: 1020.

36. Greiner KA, Murray JL, Kallail KJ. Medical student interest in alternative medicine. J Altern Complement Med. 2000; 6: 231-234.

37. Teixeira MZ, Lin CA, Martins MA. Homeopathy and acupuncture teaching at Faculdade de Medicina da Universidade de São Paulo: the undergraduates' attitudes. Sao Paulo Med J. 2005; 123: 77-82.

38. Kemper KJ, Vincent EC, Scardapane JN. Teaching an integrated approach to complementary, alternative, and mainstream therapies for children: a curriculum evaluation. J Altern Complement Med. 1999; 5: 261-268.

39. Rosenbaum ME, Nisly NL, Ferguson KJ, Kligman EW. Academic physicians and complementary and alternative medicine: an institutional survey. Am J Med Qual. 2002; 17: 3-9.

40. Cardini F, Weixin H. Moxibustion for correction of breech presentation: a randomized controlled trial. JAMA. 1998; 280: $1580-1584$.

41. Harris WS, Gowda M, Kolb JW, et al. A randomized, controlled trial of the effects of remote, intercessory prayer on outcomes in patients admitted to the coronary care unit. Arch Intern Med. 1999; 159: 2273-2278. [Erratum published in Arch Intern Med. 2000; 160: 1878.

42. Dubey NP. Integrated medicine - many approaches, one service. World Health Forum. 1997; 18: 56-58.

43. Marcus DM. How should alternative medicine be taught to medical students and physicians? Acad Med. 2001; 76: 224-229.

44. Sampson $\mathrm{W}$. The need for educational reform in teaching about alternative therapies. Acad Med. 2001; 76: 248-250.

45. Murdoch-Eaton D, Crombie H. Complementary and alternative medicine in the undergraduate curriculum. Med Teach. 2002; 24: 100-102.

46. Maizes V, Schneider C, Bell I, Weil A. Integrative medical education: development and implementation of a comprehensive curriculum at the University of Arizona. Acad Med. 2002; 77: 851-860.

\section{Conflito de Interesse}

Não declarado.

\section{Endereço para correspondência}

Rua Teodoro Sampaio, 352 - CJ 128

05406-000 - São Paulo - SP

e-mail: marcus@homeozulian.med.br 